\title{
EDITORIAL
}

\section{Can we get the necessary clinical trials in children and avoid the unnecessary ones?}

\author{
Kalle Hoppu
}

Received: 24 April 2009/Accepted: 26 May 2009/Published online: 12 June 2009

(C) Springer-Verlag 2009

Clinical trials are necessary to provide data on the safety, efficacy and optimal use of medicines. In children, data are needed to cover all ages, from newborns to adolescents. Getting the necessary clinical trials performed is required to achieve the goals of the U.S., EU and WHO paediatric medicines initiatives [1]. However, as stated in the EU Paediatric Regulation [2], unnecessary trials should be avoided for obvious ethical reasons.

For new medicinal products of therapeutic interest for children, the EU requirement of early submission of a Paediatric Investigation Plan (PIP) for approval by the Paediatric Committee (PDCO), and compliance with the PIP to get the substantial reward/incentive are likely to capture most of the necessary trials being [2] performed. So far the majority of the 122 PIPs approved by the PDCO [3] include a deferral for the studies to be performed after more data become available in adults. However, it is already evident that clinical trials of off-patent products will not be performed in children to the same extent as they will be for the new products. Only 13 (4\%) of the 313 PIPs submitted were for off-patent products [3]. When we consider that $70-80 \%$ of approved medicines are not labelled for children, and about half of the medicines used for children are off-label, the work ahead is substantial before the necessary clinical trials are performed and paediatric data have been appropriately assessed. Prioritising the medicines

K. Hoppu $(\bowtie)$

Poison Information Centre, Helsinki University Central Hospital, P.O. Box 790, Tukholmankatu 17, 00029 HUS, Helsinki, Finland

e-mail: kalle.hoppu@hus.fi

\section{K. Hoppu}

Hospital for Children and Adolescents and Dept. of Clinical Pharmacology, University of Helsinki,

Helsinki, Finland to be studied on the basis of children's needs has proven difficult. It is also uncertain whether the way the EU is funding the study of the prioritised medicines will result in necessary or 'scientifically interesting' trials.

Much paediatric data on medicines used off-label in children exist but have never been submitted for regulatory assessment and are not included in the product information. The recently published paper of Tafuri et al. in this journal [4] demonstrated that for two of the five proton pump inhibitors (PPI) used in the treatment of GERD in children, much of the necessary clinical evidence already exists and is publicly available. Existing data may also fill at least a significant part of the regulatory requirements for paediatric labelling, which is not necessarily surprising as three of the five products studied are already labelled for children in the U.S. Studying the other two PPIs is hardly necessary. However, as the example of SSRI studies in adolescents' showed [5], important existing data are not always available, particularly not data from industry-sponsored trials that show negative results for safety or efficacy [5].

The provisions of the EU Paediatric Regulation [2] for making information on all paediatric clinical trials, including results, publicly available and the requirement for the marketing authorisation holders to submit all paediatric studies, including those not in the public domain, for assessment to the competent authority should further help reduce unnecessary duplication of trials.

Available data are not always as good as for the PPIs but may still contain important information that should be considered before new clinical trials are planned. Old data, modern understandings of developmental pharmacology and modelling methods combined with clinical experience may be substituted for the learning phase of the learningconfirming cycle of trials to attain the necessary data for safety and efficacy. This approach is currently being tested 
by the WHO to bridge research gaps and to develop new formulations of tuberculosis medicines for children [6].

In the end, existing data may still have gaps. In the case of PPIs, the gap, as pointed out by de Wildt and Knibbe [7], is in children below the age of 1 year. This youngest and developmentally most different age group is generally the one where clinical trials are most necessary and most challenging, and unfortunately also less likely to be performed.

\section{References}

1. Hoppu K (2008) Paediatric clinical pharmacology-at the beginning of a new era. Eur J Clin Pharmacol 64(2):201-5

2. EC (2006) Regulation (EC) no 1901/2006 of the European Parliament and of the Council on Medicinal Products for Paediatric
Use and amending Regulation (EEC) no 1768/92, Directive 2001/ 20/EC, Directive 2001/83/EC and Regulation (EC) no 726/2004, $\mathrm{EC} / 190$

3. EMEA (2009) Press release from the PDCO March meeting. http:// www.emea.europa.eu/pdfs/human/pdco/11830509en.pdf. Accessed 13 March 2009

4. Tafuri G, Trotta F, Leufkens HG, Martini N, Sagliocca L, Traversa G (2009) Off-label use of medicines in children: can available evidence avoid useless paediatric trials? The case of proton pump inhibitors for the treatment of gastroesophageal reflux disease. Eur J Clin Pharmacol 65(2):209-216

5. Anonymous (2004) Editorial: depressing research. Lancet 363 (9418): 1335

6. Hill S, Regondi I, Grzemska M, Matiru R (2008) Children and tuberculosis medicines: bridging the research gap. Bull World Health Org 86(9):658

7. de Wildt S, Knibbe C. Knowledge of developmental pharmacology and modeling approaches should be used to avoid useless trials in children. Eur J Clin Pharmacol. doi:10.1007/ s00228-009-0655-2 